\title{
Mathematical and numerical optimality of non-singular fractional approaches on free and forced linear oscillator
}

https://doi.org/10.1515/nleng-2020-0028

Received Aug 22, 2019; accepted Nov 19, 2020.

\begin{abstract}
The prediction of oscillators is usually employed in various industrial and technological problems; such as car shock absorbers, bungee jumping, earthquake-proof buildings, musical instruments, metronome and the process of hearing. This manuscript investigates the effects of newly presented fractional operators on free and forced linear oscillators. The second order nonlinear classical governing differential equation of Duffing oscillator is reduced into second order linear classical governing differential equation of free and forced linear oscillators by invoking non-integer order differential operators namely Atangana-Baleanu and Caputo-Fabrizio. The fractionalized differential equation is solved by invoking Laplace transform method for finding the optimal solutions of displacement based on infinite series approach. The solutions of displacement are obtained via Atangana-Baleanu and Caputo-Fabrizio differential operators separately then expressed in terms of elementary and gamma functions. Finally the parametric analysis is depicted graphically on the basis of comparison of modern fractional operators subject to the emerging rheological parameters.
\end{abstract}

Keywords: free and forced linear oscillator, AtanganaBaleanu and Caputo-Fabrizio differential operators, Laplace transform, gamma and elementary functions, parametric analysis

\footnotetext{
*Corresponding Author: Kashif Ali Abro, Institute of Ground Water Studies, Faculty of Natural and Agricultural Sciences, University of the Free State, South Africa

Department of Basic Sciences and Related Studies, Mehran University of Engineering and Technology, Jamshoro, Pakistan, E-mail: kashif.abro@faculty.muet.edu.pk

Sania Qureshi, Department of Basic Sciences and Related Studies, Mehran University of Engineering and Technology, Jamshoro, Pakistan

Abdon Atangana, Institute of Ground Water Studies, Faculty of Natural and Agricultural Sciences, University of the Free State, South Africa

Department of Medical Research, China Medical University Hospital, China Medical University, Taichung, Taiwan
}

\section{Introduction}

There is no denying fact that the fractional calculus has occupied much space in the field of research because of its very impressive and fruitful properties among collection of the memory properties at each and whole of the domain in scientific problems. This idea had been originated in 1690 when a frequent and vivid question was arisen in research community as "What would happen if $\frac{d^{n}}{d x^{n}}$ if $n=\frac{1}{2}$. This question led the foundation among different fractional operators [1-12]. Riemann Livoilli fractional operator was the first fractional operator which was used by the prominent scholar namely Abel in various problems. The Riemann Livoilli fractional operator could not become a powerful and systematic tool due to some properties; such as this differential operator few has the shortcomings like "zero for the constant" and "initial and boundary conditions". But such shortcomings were overcome by Caputo fractional differential operator. Meanwhile, Caputo fractional differential operator has the singular kernel which is most important factor among fractional differential operators [13-18]. Furthermore recently, the problem of singular kernel was solved by the newly presented fractional differential operator namely Caputo Fabrizio differential fractional operator. This operator is based on non-singular exponential kernel has an ability to collect memory effects at every scattering point which are applicable on the time differences [19-28]. In this continuity, very recently a South African young mathematician namely Atangana Baleanu presented a definition of differential fractional operator consisting upon non-singular and non-local kernel. The kernel of this fractional differential operator is Mittage-Leffler function which as capability of collecting the memory effects at scattering points on the time differences as well as on the space differences [2935]. The main novelty of Atangana Baleanu fractional operator is to yield some new characteristics to narrate the complicated dynamics of physical problems with memory effects based on non-locality and non-singularity [3643]. Mickens [44] employed an explicit scheme of numeric on Duffing harmonic oscillator to generate periodic solu- 
tions. Here a good estimate for the angular frequency and harmonic balance were observed by two non-standard finite difference schemes with various symmetries. Cao et al. [45] presented numerical solutions of Duffing system via fourth order Runge-Kutta method to evlaute chaotic based on periodicity or non-periodicity. They modeled governing equation has been fractionalized for the simulation in Matlab/Simulink. Shen et al. [46] investigated fractional approach on the resonance of Duffing oscillator through the averaging method. They presented extreme good results for viscous damping, and linear stiffness by comparing analytical solutions with numerical solutions with fractional approach. In this connection, the same authors also worked on the comparative analysis of two types of fractional approaches on Duffing and van der Pol oscillators [47, 48]. Hasrati et al. [49] explored the impacts of nonlinear free and forced vibration problem by employing differential quadrature method and time periodic discretization technique. They analyzed the effects of embedded parameters on the nonlinear vibration behavior of cylindrical shells. The dynamical properties of fractional-order nonlinear oscillator have been checked for stability and feasibility of incremental harmonic balance by Shen et al. [50]. They highlighted verification of higher precision and correctness by comparing the numerical solutions with approximate analytical solutions. They also presented graphical illustrations for control parameters for dynamical responses. Motivating by above research domain, our aim is to investigate the effects of newly presented fractional operators on free and forced linear oscillators. The second order nonlinear classical governing differential equation of Duffing oscillator is reduced into second order linear classical governing differential equation of free and forced linear oscillators by invoking non-integer order differential operators namely Atangana-Baleanu and Caputo-Fabrizio. The fractionalized differential equation is solved by invoking Laplace transform method for finding the optimal solutions of displacement based on infinite series approach. The solutions of displacement are obtained via Atangana-Baleanu and Caputo-Fabrizio differential operators separately then expressed in terms of elementary and gamma functions. Finally the parametric analysis is depicted graphically on the basis of comparison of modern fractional operators subject to the emerging rheological parameters.

\section{Fractional modeling of Duffing oscillators}

The nonlinear behavior and jump phenomenon has become a classical paradigm remarkably due to Duffing oscillators. The Duffing equation is a well-known time dependent second order nonlinear differential equation of applied science. The main role of free and forced the Duffing oscillators is to disclose the hidden phenomenon of many important practical applications; for instance, nonuniformity caused by an infinite domain, prediction of diseases, orbit extraction, hardening stiffness, stochastic excitations, self-sustained vibration of a clarinet reed, nonlinear mechanical oscillators and several others. This important equation was introduced by Lord Rayleigh in his famous paper in acoustics [51]. The Duffing equation with external forcing and damping in the form of nondimensional parameters as described as

$$
\frac{d^{2} y}{d t^{2}}+2 \tau_{0} \frac{d y}{d t}+\lambda y^{3}+y-F \cos (\omega t)=0,
$$

For the Duffing equation, the letting parameters are written for their rheological deformations as $y$ represents the displacement, $t$ is time, $\tau_{0}$ symbolizes for damping ratio, $\lambda$ denotes cubic stiffness parameter, $F$ describes excitation amplitude and $\omega$ states excitation frequency. Here this manuscript is focused for free and forced behavior exhibited by the Duffing oscillator, due to these factors a parameter $\lambda=0$ which reduced the governing equation of Duffing oscillator to the forced linear oscillator. While a parameter $F=0$ then governing equation of Duffing oscillator is to be free linear oscillator. The significance of free and forced Duffing oscillator lie in the notions of resonance, phase response, amplitude response and some other aspects of the global system dynamics. Now setting the nonlinear term to zero in Eq. (1), the governing equation for free and forced responses of the linear oscillator is

$$
\frac{d^{2} y}{d t^{2}}+2 \tau_{0} \frac{d y}{d t}+y-F \cos (\omega t)=0,
$$

Subject to imposed initial conditions on the governing equation for free and forced responses of the linear oscillator as defined in Eq. (3)

$$
y(0)=0, \frac{d y(0)}{d t}=0 .
$$

In order to develop the fractional versions of governing equation for free and forced responses of the linear oscillator, we define here the newly presented fractional differentiations so called Atangana-Baleanu and Caputo- 
Fabrizio fractional operator as:

$\frac{d^{\eta_{1} \aleph(t)}}{d t^{\eta_{1}}}=\int_{0}^{t} \frac{\aleph^{\prime}(t)}{\left(1-\eta_{1}\right)} E_{\eta_{1}}\left(\frac{-\eta_{1}(z-t)^{\eta_{1}}}{1-\eta_{1}}\right) d t, \quad \eta_{1} \in[0,1]$,

$\frac{d^{\eta_{2}}(t)}{d t^{\eta_{2}}}=\int_{0}^{t} \frac{\aleph^{\prime}(t)}{1-\eta_{2}} \exp \left(\frac{-\eta_{2}(z-t)}{1-\eta_{2}}\right) d t . \quad \eta_{2} \in[0,1]$,

$$
M(1)=M(0)=1
$$

are called the normalized function for equations (4-5). Equations (4) and (5) are so called modern non-integer order differential operators of Atangana-Baleanu [52] and Caputo-Fabrizio [53]. Employing equations (4) and (5) into Eq. (2), we have fractional governing equation for free and forced responses of the linear oscillator as written below:

$$
\begin{aligned}
& \frac{d^{2 \eta_{1}} y(t)}{d t^{2 \eta_{1}}}+2 \tau_{0} \frac{d^{\eta_{1}} y(t)}{d t^{\eta_{1}}}+y(t)-F \cos (\omega t)=0, \\
& \frac{d^{2 \eta_{2}} y(t)}{d t^{2 \eta_{2}}}+2 \tau_{0} \frac{d^{\eta_{2}} y(t)}{d t^{\eta_{2}}}+y(t)-F \cos (\omega t)=0 .
\end{aligned}
$$

\section{Free and forced responses of the linear oscillator via Caputo-Fabrizio appraoch}

In order to have displacement with respect to time, the powerful and systematic technique of Laplace transform is invoked on Eq. (6) and (3), the resultant expression is obtained as

$$
\frac{q^{2} \zeta_{1} \bar{y}(q)}{\left(q+\eta_{1} \zeta_{1}\right)^{2}}+\frac{2 \tau_{0} q \zeta_{1} \bar{y}(q)}{q+\eta_{1} \zeta_{1}}+\bar{y}(q)-\frac{F q}{q^{2}+\omega^{2}}=0
$$

Here, $\zeta_{1}=\frac{1}{1-\eta_{1}}$, simplifying Eq. (8) for vivid expression of displacement, we get

$$
\bar{y}(q)=
$$

$$
\frac{F q\left(q^{2}+2 \eta_{1} \zeta_{1} q+\eta_{1}^{2} \zeta_{1}^{2}\right)}{\left(q^{2}+\omega^{2}\right)\left\{2 q^{2}+q\left(2 \tau_{0}+2 \eta_{1} \zeta_{1}\right)+\left(\zeta_{1}^{2}+2 \tau_{0} \eta_{1} \zeta_{1}+\eta_{1}^{2} \zeta_{1}^{2}\right)\right\}},
$$

Writing Eq. (9) into suitable equivalent form by using letting parameters, we arrived at

$$
\bar{y}(q)=\frac{F q}{\left(q^{2}+\omega^{2}\right)}\left[\frac{q^{2}}{\left(q-q_{1}\right)\left(q-q_{2}\right)}+\frac{2 \eta_{1} \zeta_{1} q}{\left(q-q_{1}\right)\left(q-q_{2}\right)}\right.
$$

$$
\left.+\frac{\eta_{1}^{2} \zeta_{1}^{2}}{\left(q-q_{1}\right)\left(q-q_{2}\right)}\right]
$$

Here, the quadratic equation is settled as

$$
\begin{aligned}
& \left\{2 q^{2}+q\left(2 \tau_{0}+2 \eta_{1} \zeta_{1}\right)+\left(\zeta_{1}^{2}+2 \tau_{0} \eta_{1} \zeta_{1}+\eta_{1}^{2} \zeta_{1}^{2}\right)\right\} \\
& =\left(q-q_{1}\right)\left(q-q_{2}\right),
\end{aligned}
$$

Inverting Eq. (10) by means of Laplace transform and using the property of convolution of Laplace transform, we have final analytical solution as

$$
\begin{aligned}
& y(t)=\int_{0}^{t} \cos \omega(t-z)\left\{\frac{F q_{2}^{2} e^{q_{2} t}}{\left(q_{2}-q_{1}\right)}-\frac{F q_{1}^{2} e^{q_{1} t}}{\left(q_{2}-q_{1}\right)}\right\} d z \\
& +\int_{0}^{t} \cos \omega(t-z)\left\{\frac{2 F \eta_{1} \zeta_{1} q_{2} e^{q_{2} t}}{\left(q_{2}-q_{1}\right)}-\frac{2 F \eta_{1} \zeta_{1} q_{1} e^{q_{1} t}}{\left(q_{2}-q_{1}\right)}\right\} d z \\
& +\int_{0}^{t} \cos \omega(t-z)\left\{\frac{F \eta_{1}^{2} \zeta_{1}^{2} e^{q_{2} t}}{\left(q_{2}-q_{1}\right)}-\frac{F \eta_{1}^{2} \zeta_{1}^{2} e^{q_{1} t}}{\left(q_{2}-q_{1}\right)}\right\} d z .
\end{aligned}
$$

The following identities have been employed in obtaining the final format of Eq. (12) as [54]

$$
\begin{aligned}
& \frac{q^{2}}{\left(q-q_{1}\right)\left(q-q_{2}\right)}=\frac{q_{2}^{2}}{\left(q_{2}-q_{1}\right)\left(q-q_{2}\right)}-\frac{q_{1}^{2}}{\left(q_{2}-q_{1}\right)\left(q-q_{1}\right)} \\
& \frac{q_{1}}{\left(q-q_{1}\right)\left(q-q_{2}\right)}=\frac{1}{\left(q_{2}-q_{1}\right)\left(q-q_{2}\right)}-\frac{1}{\left(q_{2}-q_{1}\right)\left(q-q_{1}\right)} \\
& \frac{1}{\left(q-q_{1}\right)\left(q-q_{2}\right)}=\frac{1}{\left(q_{2}-q_{1}\right)\left(q-q_{2}\right)}-\frac{1}{\left(q_{2}-q_{1}\right)\left(q-q_{1}\right)}
\end{aligned}
$$

Eq. (12) is the final solution of displacement with respect to time via Caputo-Fabrizio fractional differential operator so called solution with non-singular kernel but with local one. It should be noted that the Eq. (12) represents the solution of displacement with respect to time with fractional approach of non-integer order $\eta_{1} \in[0,1]$ but one can retrieve the same solution for ordinary differential operator by letting $\eta_{1}=1$.

\section{Free and forced responses of the linear oscillator via Atangana-Baleanu approach}

In order to have displacement with respect to time, the powerful and systematic technique of Laplace transform is invoked on Eq. (7) and (3), the resultant expression is obtained as

$$
\frac{q^{2 \zeta_{2}} \zeta_{2} \bar{y}(q)}{\left(q^{2 \zeta_{2}}+\eta_{2} \zeta_{2}\right)^{2}}+\frac{2 \tau_{0} \zeta^{\zeta_{2}} \zeta_{2} \bar{y}(q)}{q^{\zeta_{2}}+\eta_{2} \zeta_{2}}+\bar{y}(q)-\frac{F q}{q^{2}+\omega^{2}}=0
$$


Here, $\zeta_{2}=\frac{1}{1-\eta_{2}}$, Simplifying Eq. (14) for vivid expression of displacement, we get

$$
\begin{aligned}
& \bar{y}(q)=\frac{F q}{\left(q^{2}+\omega^{2}\right)} \\
& {\left[\frac{q^{2 \eta_{2}}\left(\zeta_{2}+2 \tau_{0}+1\right)+q^{\eta_{2}}\left(2 \zeta_{2} \eta_{2} \tau_{0} 2 \zeta_{2} \eta_{2}\right)+\eta_{2}^{2} \zeta_{2}^{2}}{\left(q^{2 \eta_{2}}+\eta_{2} \zeta_{2}\right)^{2}}\right],}
\end{aligned}
$$

Writing Eq. (15) into suitable equivalent form by using standard series, we arrived at

$$
\begin{gathered}
\bar{y}(q)=\frac{F\left(\zeta_{2}+2 \tau_{0}+1\right) q}{\left(q^{2}+\omega^{2}\right)} \sum_{m=0}^{\infty} \frac{\left(-\zeta_{2} \eta_{2}\right)^{-1} \Gamma(m+2)}{m ! \Gamma(2) q^{2 \eta_{2} m+2 \eta_{2}}} \\
+\frac{F\left(2 \zeta_{2} \eta_{2} \tau_{0} 2 \zeta_{2} \eta_{2}\right) q}{\left(q^{2}+\omega^{2}\right)} \sum_{m=0}^{\infty} \frac{\left(-\zeta_{2} \eta_{2}\right)^{-1} \Gamma(m+2)}{m ! \Gamma(2) q^{2 \eta_{2} m+3 \eta_{2}}} \\
+\frac{F \eta_{2}^{2} \zeta_{2}^{2} q}{\left(q^{2}+\omega^{2}\right)} \sum_{m=0}^{\infty} \frac{\left(-\zeta_{2} \eta_{2}\right)^{-1} \Gamma(m+2)}{m ! \Gamma(2) q^{2 \eta_{2} m+4 \eta_{2}}}
\end{gathered}
$$

Here, the following series is imposed on Eq. (16) as

$$
(p+q)^{-n} \sum_{m=0}^{\infty} \frac{(1)^{-1} \Gamma(m+n)}{m ! \Gamma(n)}(p)^{-n-m}(q)^{m},
$$

Inverting Eq. (17) by means of Laplace transform and using the property of convolution of Laplace transform, we have final analytical solution as

$$
\begin{gathered}
y(t)=F\left(\zeta_{2}+2 \tau_{0}+1\right) \int_{0}^{t} \cos \omega(t-z) \\
\sum_{m=0}^{\infty} \frac{\left(-\zeta_{2} \eta_{2}\right)^{m} \Gamma(m+2) t^{2 \eta_{2} m+2 \eta_{2}-1}}{m ! \Gamma(2) \Gamma\left(2 \eta_{2} m+2 \eta_{2}\right)} d z+F\left(2 \zeta_{2} \eta_{2} \tau_{0 \_} 2 \zeta_{2} \eta_{2}\right) \\
\times \int_{0}^{t} \cos \omega(t-z) \sum_{m=0}^{\infty} \frac{\left(-\zeta_{2} \eta_{2}\right)^{m} \Gamma(m+2) t^{2 \eta_{2} m+3 \eta_{2}-1}}{m ! \Gamma(2) \Gamma\left(2 \eta_{2} m+3 \eta_{2}\right)} d z \\
+F \eta_{2}^{2} \zeta_{2}^{2} \int_{0}^{t} \cos ? \omega(t-z) \\
\times \sum_{m=0}^{\infty} \frac{\left(-\zeta_{2} \eta_{2}\right)^{m} \Gamma(m+2) t^{2 \eta_{2} m+4 \eta_{2}-1}}{m ! \Gamma(2) \Gamma\left(2 \eta_{2} m+4 \eta_{2}\right)}
\end{gathered}
$$

Eq. (18) is the final solution of displacement with respect to time via Atangana-Baleanu fractional differential operator so called solution with non-singular kernel as well as no-local kernel. It should be also be noted that the Eq. (18) represents the solution of displacement with respect to time with fractional approach of non-integer order $\eta_{2} \in[0,1]$ but one can retrieve the same solution for ordinary differential operator by letting $\eta_{2}=1$. It is also observed that the displacement with respect to time is obtained via Atangana-Baleanu and Caputo-Fabrizio fractional differential operators that satisfy the imposed conditions.

\section{Results with parametric conclusion}

Some characteristics of free and forced linear oscillator's behaviors have been explored based on newly presented fractional operators (Atangana-Baleanu and Caputo-Fabrizio fractional differential operators). The analytical solutions have been established by invoking Laplace transform technique on the fractional governing differential equations of free and forced linear oscillator. The qualitative changes and few aspects of the global dynamics of the problem have been discussed graphically and observed to meet with physical insights. More precisely, the free and forced oscillator displayed softening and hardening performance which reflects the sign of the linearity with suitable damping ratio. The overall outcome is discussed graphically with two types of fractional operator's subject to highlight the effects as (fractal structure, plethora dynamics, primary and secondary resonances, heteroclinic and few others):

\subsection{Effects of damping ratio on free linear oscillator}

Damping discloses that how oscillations in a system decay after a disturbance or the reduction of oscillations. Such disturbance lies in the several engineering problems for instance, resistance in electronic oscillators, viscous drag in mechanical systems, scattering of light in optical oscillators and few others. Here, Figure 1 elucidates a rate at which energy is being dissipated from the free and forced oscillator or to characterize the frequency response. In our case, Figure 1 is prepared for free linear oscillator in which forcing terms is neglected. The displacement with respect to time is shown in Figure 1 by adopting two different fractional techniques namely AtanganaBaleanu and Caputo-Fabrizio fractional differential operators with varying values of damping ratio. It is clear from Figure 1 that displacement obtained via Atangana-Baleanu fractional operator does not possess resonant oscillations as expected. On the contrary, Caputo-Fabrizio fractional operator has shown a sensitive dynamics within independent amplitude of linear system. From practical point of 
view, the fractional differentiations have generated vivid results based on the jump phenomena and softening effect which bend the amplitude-frequency in overall prediction of free linear oscillator.
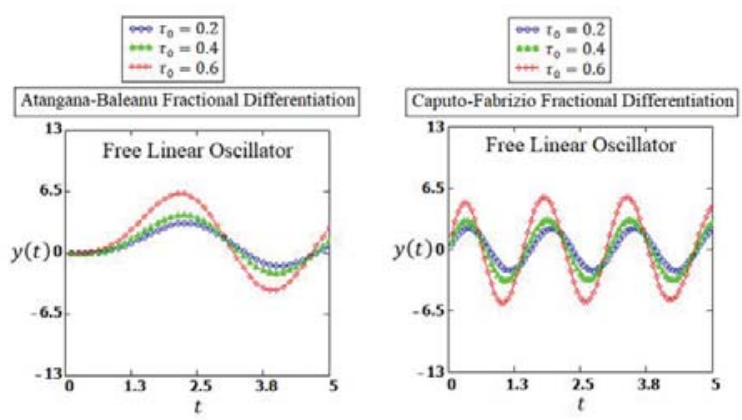

Figure 1: Profile of displacement for varying damping ratio response curves with parametric values $F=0, \omega=1.2, t=2 \mathrm{~s}, \eta_{1}=\eta_{2}=$ 0.7 subject to Atangana-Baleanu and Caputo-Fabrizio fractional differential operators.

\subsection{Effects of damping ratio on forced linear oscillator}

The forced linear oscillator is applied in a broad variety of engineering applications like driving a galvanometer, damping of oscillating gas bubbles, ultraharmonic energy harvesting, aerodynamic forces and many others. Figure 2 presents the displacement with respect to time based on forced linear oscillator via Atangana-Baleanu and CaputoFabrizio fractional differential operators with varying values of damping ratio. Based on this Figure 2, the maximum value of damping ratio has the close proximity between the both fractional approaches. Furthermore, the bending amplification of the amplitude-frequency curve is more severe in comparison of Atangana-Baleanu and Caputo-Fabrizio fractional differential operators. Therefore, the fractionalorder derivative becomes a useful tool for knowing the entire memory effects over the whole boundary of the problems. In is worth noted that the system increases its damping capacity due to fractional operator that provides a nonlocal effect of dissipation of energy.

\subsection{Effects of excitation amplitude on free and forced linear oscillator}

It is an established fact that propagation of waves strongly depends upon quantitative amplitude (maximum devia-
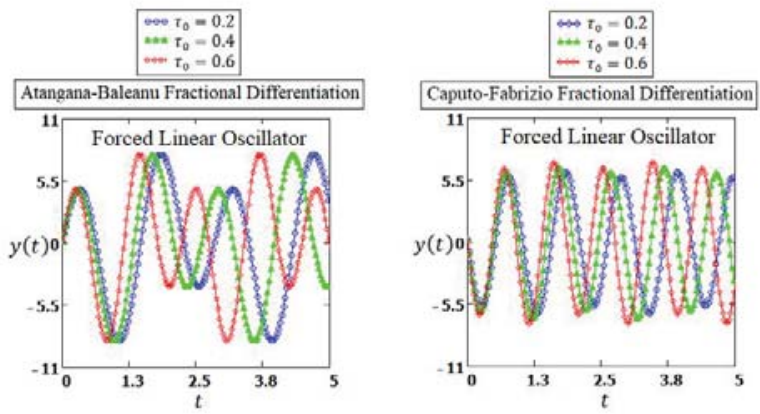

Figure 2: Profile of displacement for varying damping ratio response curves with parametric values $F=0.3, \omega=1.2, t=2 \mathrm{~s}, \eta_{1}=\eta_{2}=$ 0.7 subject to Atangana-Baleanu and Caputo-Fabrizio fractional differential operators.

tion of displacement). The role of excitation of amplitude is not only important in broadcast radio and air-band voice communications but also it is highly useful process in digital as well as analogue transmissions. Figure 3 has unified an interesting analogy namely carrier behavior of displacement based on Atangana-Baleanu and CaputoFabrizio fractional differential operators. It is clear from Figure 3 that the modulated behavior of displacement has been observed via Caputo-Fabrizio fractional differential operator which reflects the higher frequency. On the contrary, displacement based on Atangana-Baleanu fractional differential operator is perceived with the measure of the strength or intensity of the wave in Figure 3 that verifies the feasibility of Atangana-Baleanu fractional differential operator with low-frequency region.
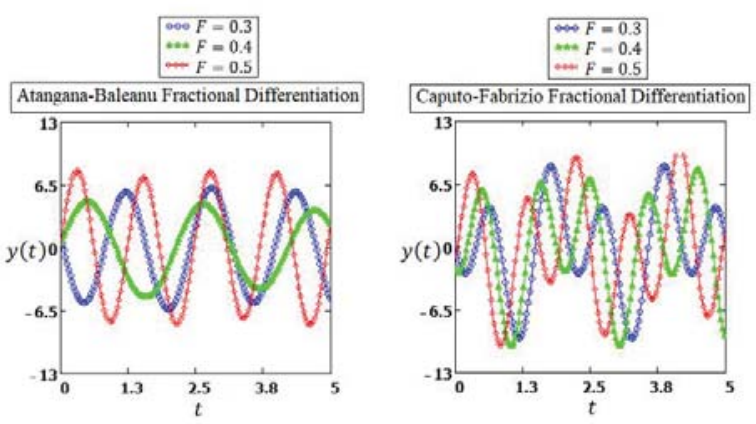

Figure 3: Profile of displacement for excitation amplitude response curves with parametric values $\omega=1.2, \tau_{0}=0.2, \eta_{1}=\eta_{2}=$ 0.7 subject to Atangana-Baleanu and Caputo-Fabrizio fractional differential operators. 


\subsection{Effects of excitation frequency on free and forced linear oscillator}

The low and high input frequencies have intensified piezoelectric and mechanical devises based on their geometries. The most of engineering systems can be controlled on ramped up frequency and ramped down frequency due to their required maintenance of energy. Here, Figure 4 shows response of excitation frequency via both fractional operators with similar bending curves which tunes the resonance frequency for vibrational analysis. The displacement obtained via Caputo-Fabrizio fractional differential operator represents weak scattering behavior and Atangana-Baleanu fractional differential operator has reciprocal behavior of displacement. From industrial application, most of industrial systems are quite dependent on scatterings of frequency for detecting the present flaws.
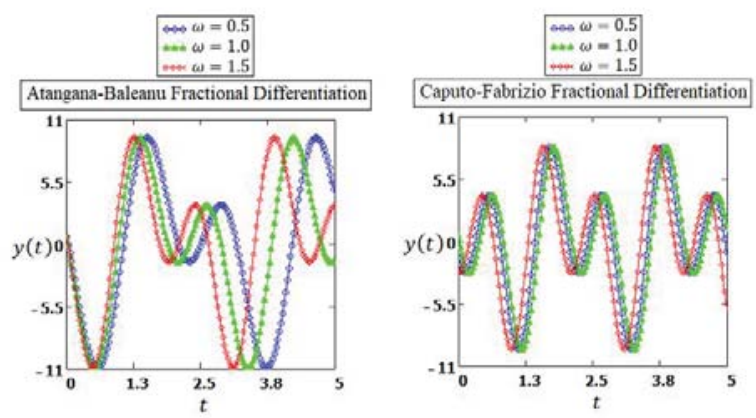

Figure 4: Profile of displacement for varying excitation frequency response curves with parametric values $\tau_{0}=0.2, F=0.3, t=$ $2 \mathrm{~s}, \eta_{1}=\eta_{2}=0.7$ subject to Atangana-Baleanu and Caputo-Fabrizio fractional differential operators.

\subsection{Comparative analysis of fractional operators on free and forced linear oscillator}

A comparison of displacement is made between AtanganaBaleanu and Caputo-Fabrizio fractional differential operators for showing the better understanding of both approaches in Figure 5. The main aim of this comparative analysis is to check the suitability and significance of Mittag-Leffler kernel and exponential kernel, both operators possess an extraordinary feature of controlling the memory effects that exist in the phenomena of free and forced linear oscillator. It is observed from Figure 5 that the displacement investigated by Atangana-Baleanu frac- tional differential operator exhibits distinct asymptotic characteristics as compared with Caputo-Fabrizio fractional differential operator. To conclude the free and forced linear oscillator model, Atangana-Baleanu fractional differential operator yields the non-local behavior of displacement with history dependence properties or memory effectiveness that can be judged from Figure 5 for intrinsic dissipative process as well.
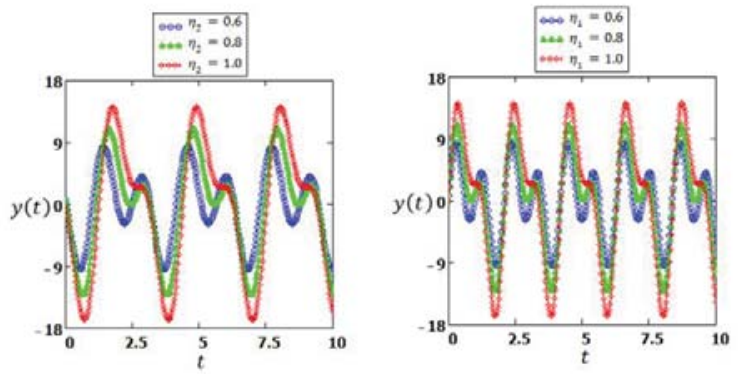

Figure 5: Comparative Analysis displacement for varying fractional parameters of Atangana-Baleanu and Caputo-Fabrizio fractional differential operators with parametric values $\tau_{0}=0.2, F=0.3, t=$ $2 s, \omega=0.5$.

Acknowledgements: The both authors are highly thankful and grateful to Mehran University of Engineering and Technology, Jamshoro, Pakistan for generous support and facilities of this research work.

Conflict of interest: The authors declare no conflict of interest regarding the publication of this paper.

\section{References}

[1] Gomez-Aguilar JF, Atangana A. New insight in fractional differentiation: power, exponential decay and Mittag-Leffler laws and applications. Eur Phys J Plus. 2017;132(1):13.

[2] Atangana A, Gomez-Aguilar JF. Hyperchaotic behaviour obtained via a nonlocal operator with exponential decay and Mittag-Leffler laws. Chaos Solitons Fractals. 2017;102:1-10.

[3] Kashif AA, Bhagwan D. A scientific report of non-singular techniques on microring resonators: an application to optical technology. Optik (Stuttg). 2020;224:165696.

[4] Qasim A, Samia R, Aziz UA, Kashif AA. Thermal investigation for electrified convection flow of Newtonian fluid subjected to damped thermal flux on a permeable medium. Phys Scr. 2020;95(11):115003.

[5] Al-Mdallal QM, Abu Omer AS. Fractional-order Legendre collocation method for solving fractional initial value problems. Appl Math Comput. 2018;321:74-84. 
[6] Abro KA. Role of fractal-fractional derivative on ferromagnetic fluid via fractal Laplace transform: A first problem via fractal-fractional differential operator. Eur J Mech BFluids. 2021;85:76-81.

[7] Atangana A, Gomez-Aguilar JF. Decolonisation of fractional calculus rules: breaking commutativity and associativity to capture more natural phenomena. Eur Phys J Plus. 2018;133(4):123.

[8] Asıf Y, Hülya D, Abro KA, Dogan K. Role of Gilson-Pickering equation for the different types of soliton solutions: A nonlinear analysis. Eur Phys J Plus. 2020;135(8):657.

[9] Atangana A, Gomez-Aguilar JF. A new derivative with normal distribution kernel: Theory, methods and applications. Physica A. 2017;476:1-14.

[10] Aziz UA, Muhammad T, Abro KA. Multiple soliton solutions with chiral nonlinear Schrödinger's equation in (2+1)dimensions. Eur J Mech BFluids. 2021;85:68-75.

[11] Jagdev S, Aydin S, Ram S, Devendra K. A reliable analytical approach for a fractional model of advection-dispersion equation. Nonlinear Eng. 2019;8(1):107-16.

[12] Kashif AA, Abdon A. Porous effects on the fractional modeling of magnetohydrodynamic pulsatile flow: an analytic study via strong kernels. J Therm Anal Calorim. 2020; DOI: https://doi.org/10.1007/s10973-020-10027-z

[13] Azhar WA, Vieru D, Fetecau C. Free convection flow of some fractional nanofluids over a moving vertical plate with uniform heat flux and heat source. Phys Fluids. 2017;29(8):082001.

[14] Abro KA, Abdon A. Numerical study and chaotic analysis of meminductor and memcapacitor through fractalfractional differential operator. Arab J Sci Eng. 2020; DOI: https://doi.org/10.1007/s13369-020-04780-4

[15] Imran MA, Khan I, Ahmad M, Shah NA, Nazar M. Heat and mass transport of differential type fluid with non-integer order time-fractional Caputo derivatives. J Mol Liq. 2017;229:67-75.

[16] Abro KA, Atangana A. A comparative analysis of electromechanical model of piezoelectric actuator through CaputoFabrizio and Atangana-Baleanu fractional derivatives. Math Methods Appl Sci. 2020;43(17):1-11.

[17] Abro KA, Abdon A. Dual fractional modeling of rate type fluid through non-local differentiation. Numer Meth Part Diff Eq. 2020.;DOI: https://doi.org/10.1002/num.22633

[18] Kashif AA, Jose FG. Role of Fourier sine transform on the dynamical model of tensioned carbon nanotubes with fractional operator. Math Methods Appl Sci. 2020; DOI: https://doi.org/10.1002/mma.6655

[19] Ozair M., Hussain T., Hussain M., Awan A.U., Baleanu D., Abro KA. A Mathematical and Statistical Estimation of Potential Transmission and Severity of COVID-19: A Combined Study of Romania and Pakistan. BioMed Res Int. 2020;2020:5607236.

[20] Saad KM, Gomez-Aguilar JF. Analysis of reaction diffusion system via a new fractional derivative with non-singular kernel. Physica A. 2018;509(1):703-16.

[21] Abro KA, Abdon A. Mathematical analysis of memristor through fractal-fractional differential operators: A numerical study. Math Methods Appl Sci. 2020;43(10):1-18.

[22] Qureshi S, Atangana A. Mathematical analysis of dengue fever outbreak by novel fractional operators with field data. Physica A. 2019;526:121127.

[23] Aziz UA, Mukarram A, Abro KA. Electroosmotic slip flow of Oldroyd-B fluid between two plates with non-singular kernel. J
Comput Appl Math. 2020;376:112885-99.

[24] Bhojraj L, Kashif AA, Abdul WS. Thermodynamical analysis of heat transfer of gravitydriven fluid flow via fractional treatment: an analytical study. J Therm Anal Calorim. 2020; https://doi.org/10.1007/s10973-020-09429-w

[25] Owolabi KM. Numerical patterns in reaction-diffusion system with the Caputo and Atangana-Baleanu fractional derivatives. Chaos Solitons Fractals. 2018;115:160-9.

[26] Saad KM, Al-Sharif EH. Analytical study for time and time space fractional Burgers' equation solutions. Adv Diff Equ. 2017:300.

[27] Kashif AA, Abdon A. A comparative study of convective fluid motion in rotating cavity via Atangana-Baleanu and CaputoFabrizio fractal-fractional differentiations. Eur Phys J Plus. 2020;135(2):226-42.

[28] Kashif AA, Ambreen S, Abdon A. Thermal stratification of rotational second-grade fluid through fractional differential operators. J Therm Anal Calorim. 2020; https://doi.org/10.1007/s10973-020-09312-8

[29] Abro KA, Atangana A. Role of non-integer and integer order differentiations on the relaxation phenomena of viscoelastic fluid. Phys Scr. 2020;95(3):035228.

[30] Bhuvnesh S, Sunil K, Paswan MK. Numerical investigation of MHD stagnation-point flow and heat transfer of sodium alginate non-Newtonian nanofluid. Nonlinear Eng. 2019;8(1):17992.

[31] Kashif AA. A Fractional and analytic investigation of thermodiffusion process on free convection flow: an application to surface modification technology. Eur Phys J Plus. 2019;135(1):31-45.

[32] Saad KM. A reliable analytical algorithm for space-time fractional cubic isothermal autocatalytic chemical system. Pramana J. Phys. 2018;91(4):51.

[33] Saad KM. AL-Sharif E. H. F., Comparative study of a cubic autocatalytic reaction via different analysis methods. Discrete and Continuous Dynamical Systems. 2019;12(3):665-84.

[34] Abro KA, Ilyas K, Jose FG. Heat transfer in magnetohydrodynamic free convection flow of generalized ferrofluid with magnetite nanoparticles. J Therm Anal Calorim. 2020; https://doi.org/10.1007/s10973-019-08992-1

[35] Kumar D, Singh J, Baleanu D, Sushila. Sushila, Analysis of regularized long-wave equation associated with a new fractional operator with Mittag- Leffler type kernel. Physica A. 2018;492:155-67.

[36] Abro KA. Fractional characterization of fluid and synergistic effects of free convective flow in circular pipe through Hankel transform. Phys Fluids. 2020;32:123102.

[37] Abro KA, Abdon A. Numerical and mathematical analysis of induction motor by means of $A B$-fractal-fractional differentiation actuated by drilling system. Numer Meth Part Diff Eq. 2020:1-15.

[38] Hadi R, Osman MS, Mostafa E, Mohammad M, Qin Z, Seyed $A B$, et al. Hyperbolic rational solutions to a variety of conformable fractional Boussinesq-Like equations. Nonlinear Eng. 2019;8(1):224-30.

[39] Kashif AA, Ambreen S, Basma S, Abdon A. Application of statistical method on thermal resistance and conductance during magnetization of fractionalized free convection flow. Int Commun Heat Mass Transf. 2020;119:104971. 
[40] Singh J, Kumar D, Hammouch Z, Ngana A. A fractional epidemiological model for computer viruses pertaining to a new fractional derivative. Appl Math Comput. 2018;316:504-15.

[41] Abro KA, Mehwish S, Abdon A, Jose FG. Thermophysical properties of Maxwell Nanoluids via fractional derivatives with regular kernel. J Therm Anal Calorim. 2020; https://doi.org/10.1007/s10973-020-10287-9

[42] Yusuf A, Qureshi S, Inc M, Aliyu Al, Baleanu D, Shaikh AA. Two-strain epidemic model involving fractional derivative with Mittag-Leer kernel. Chaos: An Interdisc J Nonlin Sci. 2018;28(12):123121. https://doi.org/10.1063/1.5074084

[43] Kashif AA, Ahmet Y. Fractional treatment of vibration equation through modern analogy of fractional differentiations using integral transforms. Iran J Sci Technol Trans A Sci. 2019;43(5):18.

[44] Mickens RE. Mathematical and numerical study of the Duffing harmonic oscillator. J Sound Vibrat. 2001;244(3):563-7.

[45] Cao J, Ma C, Xie H, Jiang Z. Nonlinear dynamics of Duffing system with fractional order damping. J Comput Nonlinear Dyn. 2010;1(4):2-6.

[46] Shen YJ, Yang SP, Xing HJ, Gao G. Primary resonance of Duffing oscillator with fractional-order derivative. Commun Nonlinear Sci Numer Simul. 2012;17(7):3092-100.

[47] Shen YJ, Yang SP, Xing HJ, Ma HX. Primary resonance of Duffing oscillator with two kinds of fractional-order derivatives. Int J Non-linear Mech. 2012;47(9):975-83.

[48] Shen Y], Wei P, Yang SP. Primary resonance of fractional-order van der Pol oscillator. Nonlinear Dyn. 2014;77(4):1629-42.

[49] Hasrati E, Ansari R, Torabi J. A novel numerical solution strategy for solving nonlinear free and forced vibration problems of cylindrical shells. Appl Math Model. 2018;53:653-72.

[50] Shen Y], Wen SF, Li XH, Yang SP, Xing HJ. Dynamical analysis of fractional-order nonlinear oscillator by incremental harmonic balance method. Nonlinear Dyn. 2016;85(3):1457-67.

[51] Strutt J. M., Scientific Papers. London: Macmillan and Co.; 1943.

[52] Atangana A, Baleanu D. New fractional derivatives with nonlocal and non-singular kernel: theory and application to heat transfer model. Therm Sci. 2016;20(2):757-63.

[53] Caputo M, Fabrizio M. A new definition of fractional derivative without singular kernel. Prog. Fract. Differ. Appl. 2015;1(2):113.

[54] Kashif AA, Imran QM, Ambreen S. Thermal transmittance and thermo-magnetization of unsteady free convection viscous fluid through non-singular differentiations. Phys Scr. 2020; https://doi.org/10.1088/1402-4896/abc981. 